\title{
Study on the Method to Improve Equipment Failure Prediction Accuracy Gang NIU ${ }^{1}$, Jie $\mathrm{GAO}^{2}$, Yin-Xia DU ${ }^{3}$ \\ ${ }^{1}$ Institute of Ordnance Technology, Hebei Shijiazhuang, 050003, China \\ ${ }^{2}$ Information College of Hebei University of Technology, Tianjin, 300401, China \\ ${ }^{3}$ Heibei College of Engineering, Hebei Shijiazhuang 050091, China \\ wbyzq@foxmail.com
}

Keywords: Failure Prediction, Accuracy, Method.

\begin{abstract}
Increasingly high degree of complexity for the equipment, resulting in the issue of failure prediction more difficult, based on the analysis of the failure prediction method, elaborated to improve the accuracy of fault prediction methods from five aspects. Finally, make a summary analysis of it.
\end{abstract}

\section{Introduction}

With the complexity of the science and technology development and application of new technologies, equipment systems increased significantly. The various components are interrelated, making the fault characteristic uncertainty, nonlinear, concurrency. Once a fault occurs not only cause significant economic losses and could endanger the safety of environmental pollution caused serious social problems [1]. Complexity of the system, mission of uncertainty, and the severity of the fault hazard makes failure prediction task has become a hot research.

\section{Analysis on Fault Forecasting Method}

At present, fault prediction methods have no uniform classification. This paper synthesizes all literature, divided fault prediction method into four main categories: Failure prediction based on model-driven technology, failure prediction technology based on expert knowledge, failure prediction based on data-driven technology and combination forecast method [2, 3, 4].

\section{Failure Prediction based on Model-driven Technology}

Failure prediction based on model-driven technology divided into two categories: Based on the physical model and based on the mathematical model. Failure prediction based on a physical model method is by analyzing the physical, chemical and other mechanisms of failure prediction, more representative is to electronic products to carry out related work; Fault prediction method based on the data model is based on the analysis of the relationship between the input, output, state prediction results are obtained, typically have a filter theory examples (such as kalman filter, filter, etc.), the forecasting methods of ARIMA, Bayesian prediction method, Markov model, etc.

\section{Failure Prediction Technology based on Expert Knowledge}

Fault prediction method based on expert knowledge was mainly used in those with no precise mathematical model or it is difficult to establish mathematical model of the system, such as the fuzzy theory and expert system. This method often rely on a certain domain experts in the field of knowledge accumulation for a long time, provides a way to solve the problem of uncertainty and complexity.

\section{Failure Prediction Based on Data-driven Technology}

A lot of practical problems, there is no economic feasible data model to describe, it is difficult to describe by expert knowledge either, only by using historical data, through certain methods by excavating the implied relationship between historical data, to achieve the purpose of fault prediction. More representative method is support vector machine and neural network, etc. This method for history data dependence degree is higher, in the case of unable to get historical data will no longer apply. 


\section{Combination Forecast Method}

Combination forecasting is the combination of different prediction methods, the advantages of comprehensive utilization of a variety of prediction, to improve the accuracy of prediction. At present, combination forecast method is more; it is an important development direction. Such as literature [5] will FMEA and the fault prediction method of combining rough set theory, documents[6] presented to the Kalman filter with a combination of expert system failure prediction method, the method described in the literature[7,8] belong combination forecasting method. Generally combination forecasting method to predict the effect of better, but the process is often more complex, combination forecasting method is still in the development process, theory is not mature enough.

\section{Improve the Accuracy of Failure Prediction Methods}

Although there are a variety of fault prediction methods, failure prediction accuracy depends not only on fault prediction method to, and affected by many factors. Here, from several angles failure prediction factors affecting the accuracy of analytical methods to improve the accuracy of the failure prediction.

\section{Depth study of Failure Mechanisms [9, 10]}

To improve the accuracy of failure prediction, we must start from the source, the failure mechanism depth analysis equipment. At present, many equipped only with fault detection capability, it can not fail to predict. Failure mechanism includes system -level and component -level fault mechanism analysis.

System-level Failure Mechanism Analysis [4].In particular, some electronic systems, although the system in a hierarchical structure, characterized in the open, to some extent, reduce the system complexity $[11,12]$. However, due to sharing of highly integrated hardware, application software can be configured to run on multiple hardware modules. When the system fails, it is difficult to determine is a hardware failure or software failure, there may be a fault generated by the interaction of both. Even worse is the combination of different hardware and software fault. In addition, with the improvement of equipment complexity, the combination between different parts of the failure mode is to be in-depth study.

Component-level Fault Mechanism Analysis. Many electronic components failure failure mechanism study is not deep, and can't fault prediction. However, some scholars put forward, the electronic device is composed of matter Can casually through the analysis of the change of its physical properties and the relationship between the faults and forecast its fault.

\section{Improve the Historical Data Acquisition Technology}

In the process of failure prediction, most of the methods depend on the historical data. Therefore, equipment history data integrity is very important to improve the forecasting accuracy. But in actual work, often lack the historical data of equipment, this brought difficulty to failure prediction. So you must improve history data acquisition techniques from the following several aspects.

Establish Scientific and Perfect Fault Related Data Collection System with the Standard. Scientific and perfect fault related data collection system and the standard is according to different failure prediction, determine the fault data types. Through artificial records or sensor monitoring means to obtain the noise as low as possible, as far as possible complete fault data, so that the fault prediction accurately and conveniently.

To Optimize the Data Acquisition Method. A large amount of historical data acquisition is the precondition of failure prediction. However, due to various reasons, led to the historical data acquisition is difficult. To some extent, limits the accuracy of fault prediction. For some devices, for example, the lack of effective monitoring means and methods, can through the development of sensor technology, internal BIT technology provides effective way for data acquisition [10].

\section{Optimize the Information Processing Method}

Under the limited conditions, fully tap available variables. On the basis of improved feature extraction methods, improve the accuracy of fault prediction.

Make Full Use of the Variable can be Detected. Using multivariate information is for fault prediction [3]. For example common based on single variable condition forecasting technology cannot 
use multidimensional vibration data of the measurement data of effective information, difficult to detect weak fault symptoms early performance degradation. Multivariable predicting can take the same time measured data of the internal relation and mutual influence between multiple variables into account.

Improve the Fault Feature Extraction Technology. Fault feature extraction is to use modern signal processing technology will signal of original feature space model with the new feature space vectors, to obtain the most effective method of characteristics. Improve the feature extraction method, the comprehensive utilization of multi-source information fusion technology, filtering technology, the time domain; frequency domain analysis technology can effectively improve the accuracy of feature extraction.

\section{Develop the Combination Forecast Algorithm}

According to different subjects should adopt different fault prediction method. In addition, because a single prediction algorithm often has certain limitation, therefore, by combining the particularity of the research object and the characteristics of forecasting methods, give full play to the advantages of each prediction method, will a variety of combined prediction algorithm, makes the optimal prediction effect.

\section{To Improve Fault Prediction Mechanism [1]}

With the development of technology, the traditional fault prediction mechanism can not meet people's needs. Development of new fault prediction mechanism is an effective way to improve the accuracy of fault prediction. Such as in-depth study of intelligent BIT Technology, the expert system, neural networks, machine learning, knowledge discovery and other intelligence theory and technology embedded into the BIT; Extensively distributed remote fault prediction methods, data sharing failure, failure prediction, experience and other information to improve forecast accuracy; PHM vigorously promote the equipment design, from system-wide point of view of the whole life failure prediction problem.

\section{Conclusion}

Modern equipment toward digital, electronic, information technology direction, structure becomes more complex, which is more demanding failure prediction method has higher accuracy requirements of its forecast. I believe that, in order to achieve a qualitative leap in failure prediction, the in-depth study of failure mechanism is the core; improved fault prediction mechanism is the key. While other methods can improve the prediction accuracy to a certain extent, in order to achieve a major breakthrough difficult.

\section{References}

[1]Zuo Xianzhang, Kang Jian, Li Hao, Tang Liwei. Overview of fault prediction technology [J]. Fire Control and Command Control, 2010.01, 35(1):1-5.

[2]Wu Mingqiang, Fang Hongzheng, Yin Dawei. Fault prognostic approach and application technique research of complex system[J]. Computer Measure and Control, 2010.18(1):70-77.

[3]Ma Jie, Xu Xiaoli, Zhou Donghua. Survey of fault predication methods for rotating machinery[J]. Process Automation Instrumentation, 2011.08, 32(8):1-3.

[4]Wang Liang, Lv Weimin, Teng Kenan, Li Jiazhe.Research on data-driven fault prediction technologies for equipments[J].Computer Measurement and Control, 2013.21(8):2087-2089, 2105.

[5]Sun Xinxin, Du Chenglie. A FMECA and rough sets linked fault prediction approach [J]. Fire Control and Command Control,2010.02,35(2):140-143,149.

[6]Huang Darong, Huang Xishu, Fan Min, Xiong Zhihua.Mixed Fault Prediction Based on Kalman Filtering and Expe rt System[J].Computer Simulation, 2005.09, 22(9):150-152.

[7]Huang J, Hu X G, Yang F. An intelligent fault diagnosis of high voltage circuit breaker based on improved EMD energy entropy and multi-class support vector machine[J].Electric Power Systems Research, 2011, 81(2): 400-407. 
[8]Widodo A, Yang B S. Machine health prognostics using survival probability and support vector machine [J]. Expert Systems with Applications, 2011, 38(1): 106-117.

[9]Cong Wei, Jing Bo.Failure prediction of Integrated avionic system[J].Electronics Optics and Control, 2013.05, 20(5):53-57,62.

[10]Deng Sen, Jing Bo.Summary of integrated diagnostics and prognostics method based on testability for electronic system[J].Control and Design, 2013.05, 28(5):641-649.

[11]Du Ningshe, Zhai Zhengjun, Wang Guoqing. Technologies of new generation avionics integration and prognostics and health management [J]. Measurement and control technology, 2011, 30( 1):1-5,9.

[12]XU Jiuping,XU Lei.Health management based on fusion prognostics for avionics system[J]. Journal of Systems Engineering and Electronics 2011,22(3): 428-436. 